\title{
On polarisation of high intensity pulses in radio pulsars
}

\author{
N. Rathnasree \\ Raman Reserach Institute, Bangalore, 560080 - INDIA \\ Joanna M. Rankin \\ University Of Vermont, Burlington, VT-05405, USA
}

\section{Introduction}

A marked anti-correlation between the degree of linear polarisation $d_{L}$ and the intensity $I$, has been reported for some pulsars (Manchester, Taylor \& Huguenin 1975; Xilouris et. al. 1994). A detailed investigation of the variation in the total polarisation of single pulses for some pulsars has been done, with proper statistical correction applied to the linearly polarised power. This study, then, reveals very different behaviour as well as some remarkable correlations in many pulsars, which reflect directly on the nature of pulsar radio emission.

The most important correction to be applied to linear polarisation data arises from the differences in the statistical properties of the Stokes parameters $Q$ and $U$, defining the linear polarisation, and the linearly polarised power $L=\sqrt{\left(Q^{2}+U^{2}\right)}$. The measured value of the linearly polarised power is larger than the true value, with the difference being $\approx \sigma \sqrt{\pi / 2}$, where $\sigma$ is the measured standard deviation in the off-pulse region in any of the Stokes parameters (Rankin, Campbell \& Spangler 1975).

High S/N individual pulses necessarily increase the system temperature, and use of the off-pulse fluctuations $\sigma_{o f f}$ underestimates the true noise fluctuations occurring during the pulse $\sigma_{o n}$. However, as $\sigma_{o n}$ increases linearly with the system temperature, it can readily be estimated as $\sigma_{o n} / \sigma_{o f f}=\left(S+S_{0}\right) / S_{0}$, where $S_{0}$ is the Jansky equivalent of the system temperature (which can be calculated from the known instrumental parameters) and $S$ is the instantaneous pulsar flux. The statistical polarization correction is then $\sigma_{\text {on }} \sqrt{\pi / 2}$.

\section{Are high intensity pulses depolarised?}

Figure. 1 shows two scatter plots, of the variation of the integrated degree of polarisation with $\mathrm{I}$. The two populations are the same data, differing only in the method of analysis. The points lying more in the top half of the figure, where $d_{L}$ falls off at higher intensities, are un-corrected. The second set of data points has been corrected statistically for linear polarisation. The behaviour of the given population with the two methods of analysis is dramatically different. 


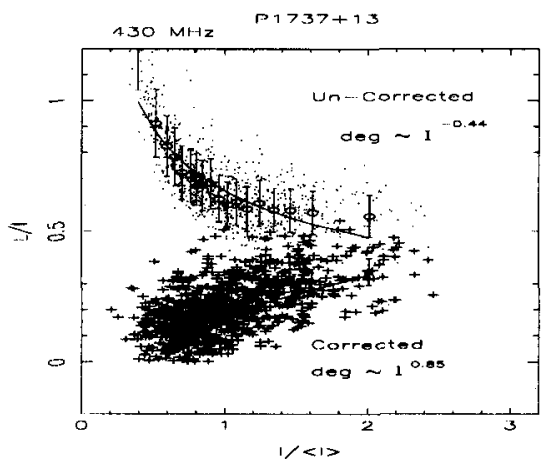

Figure 1. Scatter plot of the integrated degree of polarisation $d_{L}=\frac{L}{I}$ versus the intensity $I$ obtained from data on PSR $1737+13$, at $430 \mathrm{MHz}$. 1000 pulses are included in the figure. The intensities are normalised with respect to the average intensity under the pulse profile. The points with $1 \sigma$ error bars are sub-averaged data points, obtained by averaging the data in such a way that each bin has an equal number $(=50)$ of data points. The fitted curves are to the raw data and the power law indices obtained from these fits are indicated.

\section{Conclusions}

The differences between the current study and the previous one arise due to the proper corrections for statistical polarisation included in the present study. The increase in the degree of polarisation in high intensity pulses could perhaps be understood if subpulses within single pulses are interpreted as fundamental relativistically beamed packets of emission from elementary emitting sites. In such an interpretation, an increase in intensity could come about either due to an increase in the number of coherent emitters, or an increase in the energies (or the value of $\gamma=1 / \sqrt{\left(1-\left(\frac{v}{c}\right)^{2}\right)}$, where $v$ is the velocity) of the emitting particles. In the latter case, following the decrease in the width of sub-pulse beams with an increase in $\gamma$, there would be less superposition of emission from neighbouring emission sites and consequently less de-polarisation. This could also explain why the increase is so mild and even absent in some cases.

Acknowledgments. We thank A. Carlow, V. Izvekova, S. Suleymanova, and K. Xilouris for help with the October 92 observing. We also acknowledge A. Deshpande, V. Radhakrishnan and K. Xilouris for helpful discussions. This work was supported by a grant from the N. S. F. (AST 89-17722). Arecibo Observatory is operated by Cornell University under contract to the N. S. F.

\section{References}

Manchester, R.N., Taylor, J.H. \& Huguenin, G.R. 1975, ApJ 196, 83.

Rankin, J. M., Campbell, D. B., \& Spangler, S. 1975, NAIC Report \#46.

Xilouris, K.M., Kramer, M.; Jessner, A. \& Wielebinski, R. 1994, A\&A 288, L17. 